a)

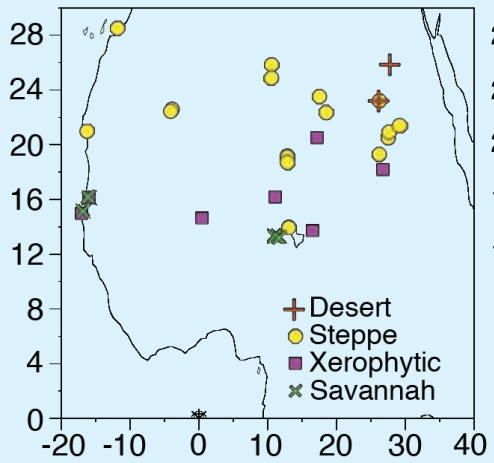

b)

$28^{\circ} \mathrm{N}$

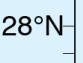

$24^{\circ} \mathrm{N}$

$20^{\circ} \mathrm{N}$

$16^{\circ} \mathrm{N}-$

$12^{\circ} \mathrm{N}-$

$8^{\circ} \mathrm{N}-$
$4^{\circ} \mathrm{N}-$
$0^{\circ}$
늋듀

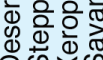

$\stackrel{\square}{\oplus} \stackrel{\oplus}{x}$

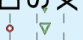

c)

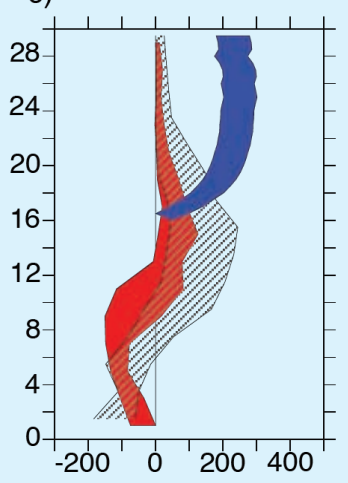

d)

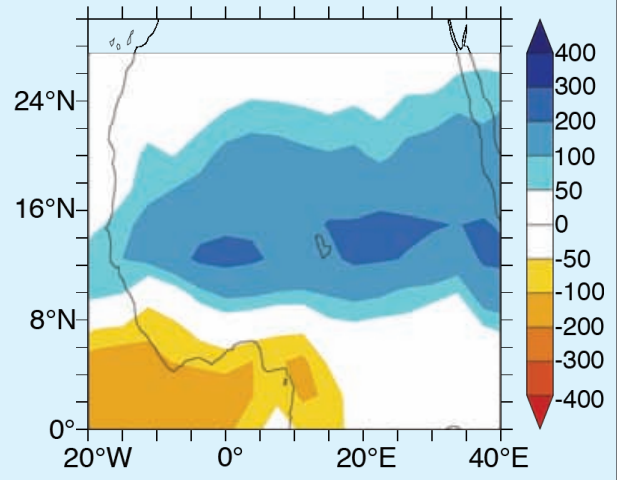

Figure 1: Biome and precipitation changes in Africa for the mid-Holocene. a) Biome distribution during the mid-Holocene. b) Zonal mean biome distribution for mid-Holocene (6kyr) and pre-industrial (Okyr) periods, and c) change in annual mean precipitation ( $\mathrm{mm} / \mathrm{yr}$ ) as simulated by the PMIP1 atmosphere alone models (red) and the PMIP2 coupled ocean-atmosphere models (black dashed). The model envelope is the range of precipitation covering $50 \%$ of the simulations around the median simulation. Blue band indicates the amount of precipitation that would be needed to replace modern desert by steppe. $\boldsymbol{d}$ ) Ensemble mean change of annual mean precipitation (mm/yr) estimated from PMIP2 coupled ocean-atmosphere simulations. These figures show that PMIP2 coupled simulations are in better agreement with data than PMIP1 atmosphere alone simulations but that they still fail to produce enough precipitation to sustain steppe as far as $23^{\circ} \mathrm{N}$, as suggested by pollen data. These figures are adapted from Jolly et al., 1998, Joussaume et al., 1999 and Braconnot et al., 2007.

results of the different models. Systematic model-data comparisons are a key element of the program. PMIP will continue to foster the development of improved methods of reconstructing climate parameters from paleo-observations, and of rigorous statistical approaches for comparing simulated and observed climates. This will ensure that these comparisons focus on appropriate variables and scales.

PMIP is now also interested in new periods, such as the early Holocene and the last glacial inception, and in new topics, such as "water-hosing experiments" (testing the sensitivity of the Atlantic meridional overturning to surface water flux forcing). State-of-the-art models can now be used to examine changes in short-term climate variability and in climate extremes, such as droughts or storms. PMIP has also started to promote the development of "forward models" for use in model evaluation and, increasingly, for coupling directly within a climate-model framework. These new challenges will require new data syntheses, including syntheses of high-resolution indicators.

PMIP has developed a new Science and Implementation Plan to help prepare for the next IPCC assessment. The plan (available from the PMIP2 website) is structured around four themes: 1 ) evaluation of Earth System models for 6 kyr BP and LGM, 2) interglacials and warm periods, 3) abrupt climate change, and 4) measures of model skill in simulating paleoclimate conditions. Details will be discussed at the next PMIP2 workshop (14-19 Sept 2008, Colorado, USA). This workshop will also showcase ongoing sub-projects and explore linkages between climate and environmental studies.

*World Climate Research Programme/Climate Variability and Predictability

\section{References}

Braconnot, P., et al., 2007: Results of PMIP2 Coupled Simulations of the Mid-Holocene and Last Glacial Maximum - Part 1: Experiments and Large-Scale Features, Climate of the Past, 3: 261-277.

Crucifix, M., Braconnot, P., Harrison, S.P. and Otto-Bliesner, B.L., 2005: Second Phase of Paleoclimate Modelling Intercomparison Project, EOS, 86: 264.

Harrison, S., Braconnot, P., Hewitt, C. and Stouffer, R.J., 2002: Fourth International Workshop of the Palaeoclimate Modelling Intercomparison Project (PMIP): Launching PMIP Phase I, EOS, 83 447-447.

Jolly, D., et al., 1998: Biome Reconstruction from Pollen and Plant Macrofossil Data for Africa and the Arabian Peninsula at 0 and $6 \mathrm{Ka}$, Journal of Biogeography, 25: 1007-1028.

Joussaume, S., et al., 1999: Monsoon Changes for 6000 Years Ago: Results of 18 Simulations from the Paleoclimate Modeling Intercomparison Project (PMIP), Geophysical Research Letters, 26 $859-862$

\title{
An overview of some current CLIVAR modeling activities
}

\section{Howard Cattle}

International CLIVAR Project Office, Southampton, UK; hyc@noc.soton.ac.uk

Modeling activities form a key component of CLIVAR, the World Climate Research Programme's Climate Variability and Predictability Project. From the global modeling perspective, these activities are carried out and coordinated through the joint WCRP/CLIVAR Working Group on Coupled Modelling (WGCM) and the CLIVAR Working Groups on Ocean Model Development (WGOMD) and on Seasonal to Interannual prediction (WGSIP). A key task for all of these groups is to maintain an oversight, and encourage developments in the component and coupled models needed for climate prediction (e.g., see Fig. 1). CLIVAR's regional monsoon and ocean basin panels also have modeling foci, often linked to results from CLIVAR-sponsored field programs such as the North American Monsoon Experiment (2004) or the current Tropical Atlantic Climate Experiment (2006-10), which has brought improved coverage of surface and subsurface data and dedicated process studies in the east-

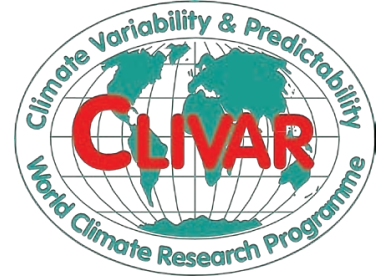

ern tropical Atlantic. In addition, CLIVAR's Global Synthesis and Observation Panel (GSOP) has a key activity to inter-compare the outputs of current global ocean synthesis efforts that use data assimilation of the historical dataset of ocean observations to produce consistent ocean analyses.

An important activity for the WGCM has been the coordination, under the Coupled Model Intercomparison project (CMIP-3), of the global coupled climate 


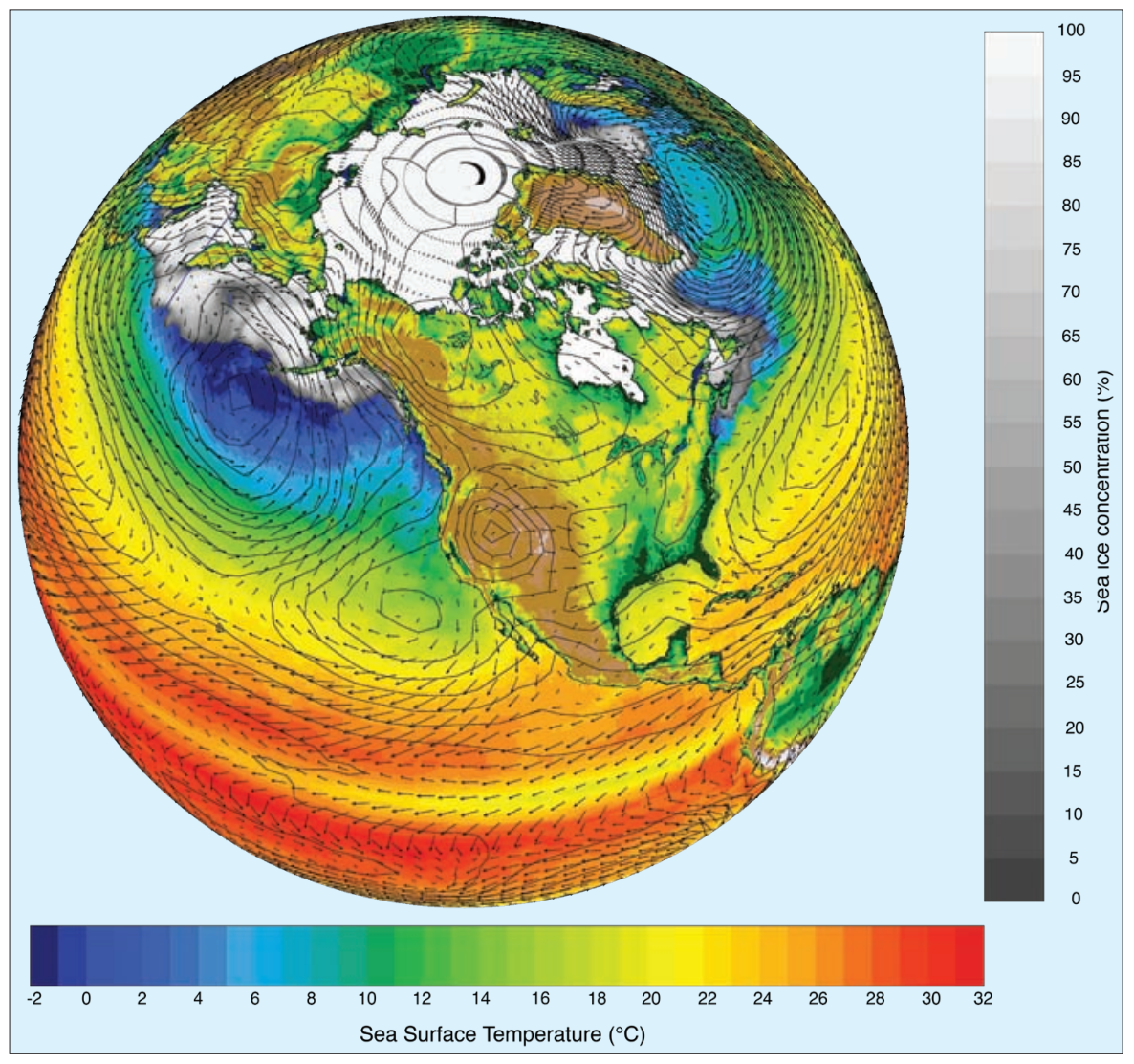

Figure 1: This figure shows, for the atmosphere, vectors depicting the winds in the lowest model layer, and the sea level pressure, shown as lines of constant pressure. The ocean model surface temperatures are shown in blue to red colors and the sea ice concentration is shown in gray. This simulation is from the Department of Energy-sponsored Parallel Climate Model (PCM). This coupled model consists of the NCAR Community Climate Model (CCM) for the atmospheric component, the Los Alamos National Laboratory Parallel Ocean Program (POP) for the ocean component, and the Naval Postgraduate School sea ice model. The PCM was specifically designed to execute on parallel supercomputers. (Figure designed by Gary Strand of NCAR, using FERRET, from PMEL).

model runs carried out in support of the IPCC AR4 WG-1 and their archiving and dissemination for the worldwide research community by the Program for Climate Model Diagnosis and Intercomparison (PCMDI) (www-pcmdi.llnl.gov/projects/ cmip/index.php). Community access and analysis of this $+35 \mathrm{~Tb}$ data archive, from 15 modeling groups across 11 countries, continues unabated. As of November 2007, more than 1000 registered users had downloaded data (over $300 \mathrm{~Tb}$ in total), and over 300 related journal articles had been published. With IGBP-AIMES, WGCM is now working to set the strategy for the next generation climate simulations that:

1. Identify new components in preparation for inclusion in atmosphere-ocean general circulation models (e.g., chemistry, aerosols, carbon cycle, dynamic vegetation).

2. Establish communication for coordination through the WCRP (WGCM and WGSIP in particular) and IGBP (AIMES in particular) and the Integrated Assessment (IA) modeling teams (the recently formed "Scenarios Consortium").

3. Propose an experimental design for $21^{\text {st }}$ century climate change experiments involving initialized short-term (decadal prediction) experiments to ca. 2030, and long-term mitigation/adaptation experiments to 2300 .
4. Specify the requirements for new stabilization scenarios (with regard to im-

Both WGCM and WGSIP are engaged in developing the protocol for the proposed near-term decadal simulations out to ca. 2030 (point 3 above). WGSIP is also coordinating the pan-WCRP Climate www.clivar.org/organization/wgsip/chfp/ chfp.php). The experiment is aimed at testing the hypothesis that there is currently untapped seasonal predictability due to interactions (and memory) among all the elements of the climate system (Atmosphere-Ocean-Land-Ice). It therefore calls for runs of seasonal prediction models that include the relevant interactions among land-ocean-atmosphere-ice. A data management plan for the project has been formulated and a call has been put out encouraging proposals for diagnostic sub-projects from the community. The CHFP was launched at the WCRP Seasonal Prediction Workshop in Barcelona in June 2007, another outcome of which was a consensus statement on the current status of seasonal prediction, available from: www.clivar.org/organization/wgsip/spw/ spw_position.php

WGOMD activities have been focused on the completion of an intercomparipacts, mitigation, and adaptation). system Historical Forecast Project (CHFP; son of 7 global ocean ice models run for 500 years using a fixed seasonal cycle of inputs to derive the atmospheric forcing. A joint paper for the peer-reviewed literature that documents the experimental design and simulation results of these CORE-I (Coordinated Ocean-ice Reference Experiment-I) integrations is in preparation. During 2008-9, WGOMD will explore the CORE-II experimental protocol, which focuses on inter-annually varying forcing based on reanalysis and observational products, aiming for a peer-reviewed paper in late 2009. CORE-III activities encompass exploration of the impact on the ocean of enhanced freshwater perturbations around the region of the Greenland ice cap. During 2008, WGOMD also plan to develop the concept of a repository for evaluating ocean simulations, giving webbased guidance on ocean model evaluation. Further information on the CORE activity can be found at www.clivar.org/ organization/wgomd/core/core.php and more information on CLIVAR in general at www.clivar.org. 